Z. klin. Chem. u. klin. Biochem.

8. Jg., S. $45-48$, Januar 1970

\title{
Wirkung von Barbital auf Enzyminduktionen in der Rattenleber
}

\author{
Von H. KRöner, H.-E. BojaR ${ }^{1}$ ), S. HollmanN und W. Staib \\ Aus dem Institut für Pbysiologische Chemie der Universität Diisseldorf
}

(Eingegangen am 1. September 1969)

\begin{abstract}
Es wird über eine akute Hemmung von Enzyminduktionen durch Barbital berichtet. Sowohl die Induktion der Tyrosin-2-OxoglutaratTransaminase und Tryptophanpyrrolase durch Cortisol als auch die Induktion der Thrconindehydratase durch Caseinhydrolysat werden gehemmt. Die Induktionshemmung durch Barbital ist noch zu einem Zeitpunkt zu erzielen, wenn Hemmstoffe der RNA-Synthese wie Aktinomycin D nicht mehr wirksam sind. Parallel zur Hemmung der Cortisol-bedingten Enzyminduktion geht eine Hemmung der Cortisolgluconeogenese durch Barbital.
\end{abstract}

\section{The action of barbital on enzyme induction in rat liver}

The acute inhibition of enzyme induction by barbital is reported. The induction of both tyrosine-2-oxo-glutarate transaminase and tryptophan pyrrolase by cortisol, and the induction of threonine dehydrase by casein are inhibited. Barbital is still active as an inhibitor in later stages of the induction when inhibitors of RNA synthesis, like actinomycin $D$, arc no longer effective. In parallel with the inhibition of induction by cortisol, barbital also inhibits cortisol gluconeogenesis.

Barbital-Natrium bewirkt kurzfristig eine Hemmung der Protein- und RNA-Synthese in der Rattenleber (1). Diese Synthesehemmung ist nicht allein mit einem durch Barbital bedingten Abfall der Körpertemperatur zu erklären, vielmehr ist der Einfluß der veränderten Körpertemperatur auf die Proteinsynthese im ,physiologischen Bereich" vergleichsweise gering (2). Eine Folge der gehemmten Proteinsynthese ist die Aktivitätsabnahme verschiedener Leberenzyme und in der Leber synthetisierter Enzyme mit kurzer biologischer Halbwertszeit $(3,1)$.

In Fortführung dieser Arbeiten erschien es uns angezeigt, die akute. Wirkung von Barbital auf Enzyminduktionen zu untersuchen. Als Modell dienten uns einerseits die Induktionen der Tryptophan-Pyrrolase (L-Tryptophan: Oxygen-Oxydoreduktase, EC 1.13.1.12) und der Tyrosin-2-Oxoglutarat-Transaminase (L-Tyrosin: 2Oxoglutarat-Aminotransferrase, EC 2.6.1.5) durch Cortisol $(4,5)$, ferner die Înduktion der Threonindehydratase (L-Threonin Hydrolỹase (desaminierend), EC 4.2.1.16) durch Hunger und Aminosäuregemische (6).

Die Cortisolinduktion ist anfänglich durch Hemmer der RNA-Synthese wie Aktinomycin D, später nur noch durch Hemmer der Proteinsynthese wie Puromycin zu blockieren (7). Wir versprachen uns daher von Versuchen zur Induktionshemmung mit Barbital Aufschluß darüber, ob Barbital die Proteinsynthese nur indirekt über eine Hemmung der RNA-Synthese oder aber direkt und primär hemmt.

Für den Zusammenhang zwischen Cortisol-bedingter Enzyminduktion und Gluconeogenese $(8,9)$ erschien ein Vergleich der Hemmung beider Prozesse durch Barbital interessant.

Wir haben daher neben den Enzymaktivitäten auch das Leberglykogen bestimmt.

1) Dissertation, Medizinische Fakultät der Universität Düsseldorf.

\section{Methodik}

Sämtliche Versuche wurden an 200 bis $300 \mathrm{~g}$ schweren männlichen Albinoratten vom Wistar II-Stamm der Firma Brünger, Bokel, durchgeführt. Soweit doppelseitig adrenalektomierte Tiere Verwendung fanden, waren diese 5 Tage vor Versuchsbeginn in leichter Äthernarkose von einem dorsalen Medianschnitt aus operiert worden. Sie erhielten 0,9 proz. $\mathrm{NaCl}$-Lösung zum Trinken und, wenn nicht anders vermerkt, Standardfutter (Firma Höveler, Langenfeld-Immingrath). Die bei einem Teil der Versuche an der Threonindehydratase erforderliche proteinfreie Diät wurde nach den Angaben von Prтot (10) hergestellt. $1 \mathrm{~kg}$ Diät enthielt $910 \mathrm{~g}$ Glucose, $50 \mathrm{~g}$ Mazolaöl, $40 \mathrm{~g}$ Salzmischung USP III (11) und Vitamine (12). 5-6 Tage lang vor Versuchsbeginn erhielten die Ratten diese proteinfreie Diät ad libitum.

Cortisol (Merck AG, Darmstadt) wurde in 0,9proz. NaCl-Lösung unter Zusatz eines Suspensionsträgers (Hoechst, Frankfurt) suspendiert und in einer Dosis von $5 \mathrm{mg} / 100 \mathrm{~g}$ Körpergewicht mittels Schlundsonde verabreicht.

Barbital (Merck AG, Darmstadt) wurde in Form des Natriumsalzes als 1,5 proz. Lösung in 0,9 proz. $\mathrm{NaCl}$-Lösung i. p. injiziert, und zwar $15 \mathrm{mg} / 100 \mathrm{~g}$ Körpergewicht.

Säurehydrolysiertes und pankreatisches Caseinhydrolysat (Merck AG, Darmstadt) wurde als 50proz. wäßr. Lösung in einer Dosis von $0,5 \mathrm{~g} / 100 \mathrm{~g}$ Körpergewicht durch Scblundsonde verabfolgt.

Die Applikation von Cortisol, Barbital-Natrium und Caseinhydrolysat erfolgte im Ätherrausch. Die Ratten wurden durch Schlag auf den Kopf getötet und aus den Bauchgefäßen entblutet. Die Leberproben für die Tryptophanpyrrolase und Threonindehydratase wurden sofort verarbeitet, die zur Bestimmung der Tyrosin-2-Oxoglutarat-Transaminase verwendeten Leberteile hingegen zunächst in flüssiger Luft tiefgefroren und dann bei $-18^{\circ} 5$ bis 6 Stdn. aufbewahrt.

Die Bestimmung der Tryptophanpyrrolase erfolgte nach der von KNox und Aurrbacr (13) beschriebenen Methode.

Die Tyrosin-2-Oxoglutarat-Transaminase wurde nach den Angaben von EwaLD und HüBENER (14) bestimmt.

Die Messung der Threonindehydratase-Aktivität erfolgte nach Pitor und Mitarbeiter (6). Diese Methode wurde dahingehend variiert, daß wir die Leber statt in der vierfachen in der neunfachen Puffermenge homogenisierten. Als Eichsubstanz für die verwendete Farbreaktion nach Gruenberg (15) diente 2-Oxobuttersäure reinst der Firma Schuchardt, München. Da diese Farbreaktion nicht spezifisch für 2-Oxobuttersäure ist, sondern allgemcin Aldehyde und Ketone erfaßt, stört der auch im $\AA$ thanol p. a. der Firma Metck AG, Darmstadt enthaltene Acetaldehyd 
$(0,0005 \%)$. Um diese Fehlerquelle auszuschalten, haben wir den Alkohol, der der besseren Löslichkeit des Hydrazons dient, erst nach der Natronlauge hinzugefügt. $\mathrm{Da}$ die Hydrazonbildung der Protonenkatalyse bedarf, kann der Acetaldehyd jetzt die Ergebnisse nicht mehr verfälschen.

\section{Ergebnisse}

Vier Stunden nach einmaliger Cortisolgabe mittels Schlundsonde ist die Aktivität der Leber-Tyrosin-2Oxoglutarat-Transaminase auf das 3,7 fache des Kontrollwertes signifikant angestiegen (Abb. 1). Eine zusätzliche intraperitoneale Injektion von Barbital-Natrium bewirkt eine je nach Zeitraum der Applikation mehr

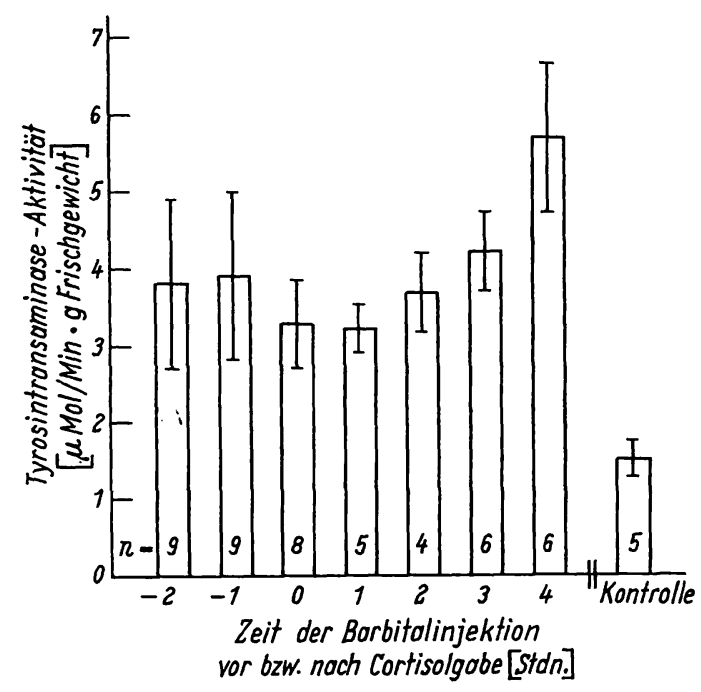

Abb. 1

Enzymaktivität der Tyrosin-2-Oxoglutarat-Transaminase in der Rattenleber nach Induktion mit Cortisol $50 \mathrm{mg} / \mathrm{kg}$ per os und zusätzlicher Injektion von Barbital-Natrium, $150 \mathrm{mg} / \mathrm{kg} \mathrm{i.} \mathrm{p.} \mathrm{Die} \mathrm{Ab-}$ szissenwerte geben die Zeiten der Barbitalinjektion vor bzw. nach gegeben. Die Zahlen in den Säulen geben die Anzahl der Versuche an, eingezeichnet ist die Standardabweichung

oder weniger ausgeprägte Hemmung der Enzyminduktion durch Cortisol. Ein oder zwei Stunden vor Cortisol verabfolgt senkt Barbital die Enzymaktivität um etwa $30 \%$. Der niedrigste Enzymspiegel resultiert aus einer gleichzeitigen Gabe von Barbital und Cortisol. Die Aktivität der Tyrosin-2-Oxoglutarat-Transaminase liegt dann um $52 \%$ niedriger als nach alleiniger Cortisolgabe. Der Aktivitätsunterschied ist mit $p<0,001$ hoch signifikant. Verabreicht man das Barbital nach dem Cortisol, ist der hemmende Effekt um so geringer, je später die Barbitalinjektion erfolgt, bleibt aber auch noch signifikant, wenn Barbital drei Stunden nach der Cortisolgabe, also eine Stunde vor dem Töten gespritzt wird $(\mathrm{p}<0,01)$.

Wird die Leber-Tryptophanpyrrolase wie die Tyrosin2-Oxoglutarat-Transaminase durch eine einmalige Cortisoldosis induziert, so erreicht ihre Aktivität nach vier Stunden das 5,3 fache des Kontrollwertes (Abb. 2). Injiziert man Barbital ein oder zwei Stunden vor dem Cortisol, ist die Aktivität um 26\% bzw. $48 \%$ ( $<<0,01)$ niedriger. Bei gleichzeitiger Verabreichung von Barbital und Cortisol zeigt die Induktion der Tryptophanpyrrolase ein Minimum, dessen Wert $48 \%$ unter dem Induktionsniveau

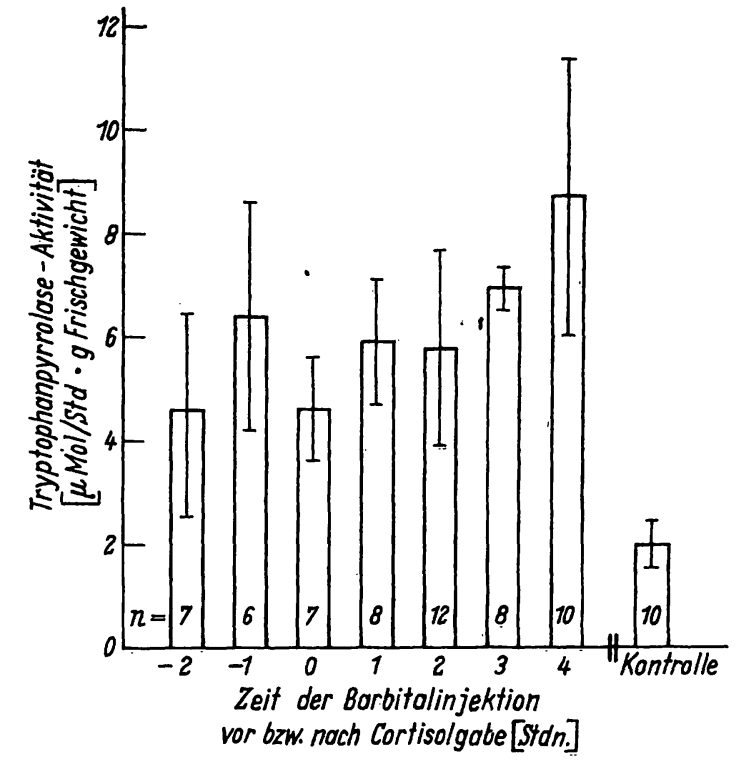

Abb. 2

Enzymaktivität der Tryptophanpyrrolase in der Rattenleber nach Enzymaktivitat der $150 \mathrm{mg} / \mathrm{kg}$ per os und zusätzlicher Injektion von Barbital-Natrium $150 \mathrm{mg} / \mathrm{kg}$ i. p. (s. Legende Aḅ. 1)

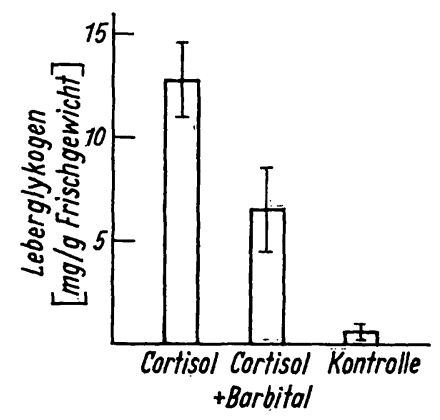

Abb. 3

Leberglykogen adrenalektomierter Ratten in $\mathrm{mg} / \mathrm{g}$ Frischgewicht nach 20 Stdn. Nahrungsentzug und Gabe von Cortisol und Barbital. Cortiso $50 \mathrm{mg} / \mathrm{kg}$ per os, Barbital-Natrium $150 \mathrm{mg} / \mathrm{kg} \mathrm{i}$. p. jeweils 6 Stdn. vor der Tötung. Mittelwerte von 6 Einzelwerten \pm Standardabweichung

gelegen, mit $\mathrm{p}<0,01$ eindeutig signifikant ist. Ein oder zwei Stunden nach dem Cortisol verabfolgt, bewirkt Barbital etwa die gleiche Hemmung der Induktion der Tryptophanpyrrolase von $32 \%(p<0,01)$. Selbst wenn das Barbital drei Stunden später als das Cortisol gespritzt wird, beobachtet man noch eine Hemmung der Cortisolinduktion um $20 \%$.

Die Cortisol-bedingte Gluconeogenese wird durch gleichzeitige Barbitalinjektion ebenfalls gehemmt (Abb. 3). 6 Stunden nach oraler Cortisolgabe an nüchterne adrenalektomierte Ratten enthält $1 \mathrm{~g}$ Leber $12,7 \mathrm{mg}$ Glykogen. Im Vergleich zu unbehandelten Kontrolltieren - $0,4 \mathrm{mg} / \mathrm{g}$ Leber - ist das eine erhebliche Zunahme. Wird gleichzeitig mit dem Cortisol Barbital injiziert, so enthält $1 \mathrm{~g}$ Leber dieser Tiere $6 \mathrm{Stdn}$. später im Mittel nur $6,5 \mathrm{mg}$ Glykogen, also fast genau $50 \%$ von dem Glykogengehalt, der nach alleiniger Cortisolbehandlung vorhanden ist. Dieser Unterschied ist statistisch hochsignifikant $(p<0,001)$.

Die Leber-Threonindehydratase ist im Gegensatz zur Tyrosin-2-Oxoglutarat-Transaminase und Tryptophanpyrrolase durch eine einmalige Cortisoldosis von $5 \mathrm{mg} /$ $100 \mathrm{~g}$ nicht kurzfristig induzierbar. Vier Stunden nach 
Verabfolgung von Cortisol mittels Schlundsonde liegt die Aktivität der Threonindehydratase sowohl bei normal ernährten adrenalektomierten Ratten als auch bei Tieren mit proteinfreier Diät im Bereich der Kontrollwerte.

Läßt man nicht-adrenalektomierte, proteinfrei ernährte Tiere hungern, so kommt es innerhalb der ersten 24 Stdn. zu keiner statistisch gesicherten Änderung des Enzymspiegels (Tab. 1). Erst nach 30 Stdn. steigt die Threonindehydratase-Aktivität signifikant auf das Fünffache der Kontrollen an $(p<0,01)$. Allerdings schwan-

Tab. 1

EinfluB von Hunger und einer einmaligen Gabe von säurehydrolysiertem Casein $(0,5 \mathrm{~g} / 100 \mathrm{~g}$ Körpergewicht) auf die Threonindehy dratase-Aktivität bei proteinfrei ernährten Ratten

\begin{tabular}{|c|c|c|}
\hline \multirow[b]{2}{*}{$\begin{array}{l}\text { Futterentzug } \\
\text { (Stdn.) }\end{array}$} & \multicolumn{2}{|c|}{$\begin{array}{l}\text { Threonindehydrataseaktivität } \\
\text { (mU/g Leber-Frischgewicht) }\end{array}$} \\
\hline & $\begin{array}{c}\text { nach der } \\
\text { Hungerperiode }\end{array}$ & $\begin{array}{l}\text { nach der Gabe } \\
\text { von Casein- } \\
\text { hydrolysat und } \\
\text { anschließender } \\
\text { Hungerperiode }\end{array}$ \\
\hline 8 & - & $\begin{aligned} 0,41 & \pm 0,08 \\
(n & =3)\end{aligned}$ \\
\hline 12 & $\begin{aligned} 0,81 & \pm 0,21 \\
(\mathrm{n} & =4)^{2}\end{aligned}$ & $\begin{array}{c}1,31 \pm 0,50 \\
(n=4)\end{array}$ \\
\hline 24 & $\begin{array}{c}0,67 \pm 0,20 \\
(n=4)\end{array}$ & - \\
\hline 30 & $\begin{array}{c}2,53 \pm 1,39 \\
(n=4)\end{array}$ & $\begin{array}{c}7,06 \pm 0,67 \\
(n=4)\end{array}$ \\
\hline Kontrollen & $\begin{array}{c}0,52 \pm 0,23 \\
(n=5)\end{array}$ & \\
\hline
\end{tabular}

ken die Werte erheblich. Während einige Ratten nach $30 \mathrm{Stdn}$. Hunger einen hohen Enzymspiegel aufweisen, bleibt bei anderen die Aktivität im Kontrollniveau.

Eine ausgeprägtere und einheitlichere Induktion des Enzyms läßt sich dadurch erzielen, daß den nicht adrenalektomierten Ratten nach 5 bis 6 Tagen proteinfreier Diät zu Beginn der Hungerperiode eine einmalige Dosis von säurehydrolysiertem Casein mittels Schlundsonde verabreicht wird (Tab. 1). Nach 30 Stdn. ist die Threonindehydratase-Aktivität auf das Dreizehnfache des Ausgangswertes erhöht $(p<0,01)$. Steiler und höher steigt die Enzymaktivität bei einer zweimaligen Gabe von pankreatischem Caseinhydrolysat an. Erhalten nicht adrenalektomierte, proteinfrei ernährte Ratten während einer $24 \mathrm{stdg}$. Hungerperiode 12 und $6 \mathrm{Stdn}$. vor dem Töten pankreatisches Caseinhydrolysat, beobachtet man einen Anstieg des Enzymspiegels auf das 38 fache des Kontrollwertes.

Wie aus Abbildung 4 hervorgeht, vermag eine zusätzliche intraperitoneale Barbitalinjektion den Aktivitätsanstieg der durch pankreatisches Caseinhydrolysat induzierten Threonindehydratase weitgehend $z u$ hemmen. Verabreicht man Barbital 3, 6 oder 9 Stdn. vor dem Töten, so erhält man im Vergleich zu nur mit Caseinhydrolysat behandelten Tieren eine signifikant erniedrigte Enzymaktivität $(p<0,01)$. Das Optimum der Barbitalwirkung mit einer Hemmung von $67 \%$ erhält man bei Injektion des Barbiturats gleichzeitig mit der zweiten Caseindosis 6 Stdn. vor der Tötung. Auch bei einer früheren oder späteren Applikation beobachtet man eine deutliche Hemmung der Threonindehydratase-

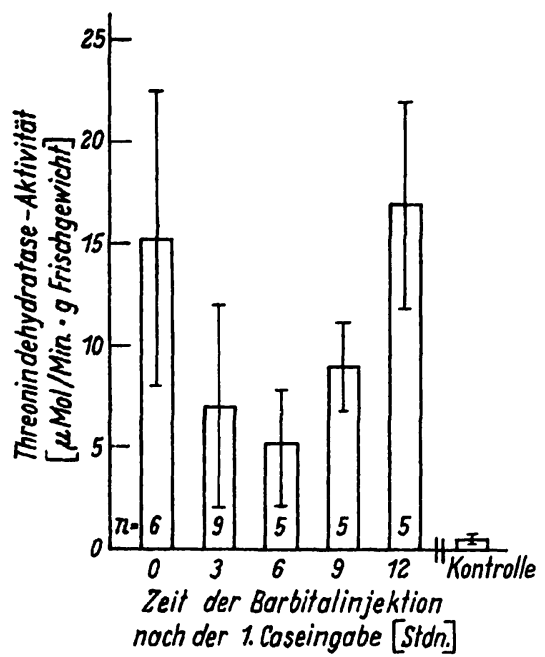

Abb. 4

Enzymaktivität der Threonindehydratase der Leber nach Induktion mit Caseinhydrolysat und zusätzlicher Gabe von Barbital-Natrium, $150 \mathrm{mg} / \mathrm{kg}$ l. p. Die Abszissenwerte geben die Zeiten der Barbitalinjektion nach der ersten Caseingabe an. Die Zahlen in den Säulen geben die Anzahl der Versuche an. Eingezeichnet ist die Standardabweichung

Induktion von $58 \%$ und $47 \%$ bei Barbitalgabe 9 bzw. 3 Stdn. vor der Tötung. Injiziert man Barbital dagegen 12 Stdn. vor dem Töten zusammen mit der ersten Caseindosis, beträgt die Aktivitätsminderung lediglich $10 \%$ und ist nicht signifikant.

\section{Diskussion}

Wie schon einleitend erwähnt, erwarteten wir von den Versuchen zur Hemmung der Enzyminduktion durch Barbital eine Bestätigung der Annahme, daß die Proteinsynthese nicht nur als Folge der RNA-Synthesehemmung, sondern primär und direkt durch Barbital gehemmt wird (1). Die Aktivitätszunahme der Tryptophanpyrrolase und der Tyrosin-2-Oxoglutarat-Transaminase der Leber nach Zufuhr von Cortisol beruht auf einer Neusynthese von Enzymprotein $(16,17,18)$. Diese Induktion läßt sich durch Hemmstoffe der RNASynthese wie Aktionomycin D nur zu einem frühen Zeitpunkt, etwa bis 1 Std. nach der Cortisolgabe stören (7). Eine spätere Hemmung der Cortisolinduktion ist nur noch über eine Hemmung der Proteinsynthese zu erzielen $(19,20)$. Die von uns gefundene Verminderung der Cortisol-bedingten Enzyminduktion durch Barbital kurz vor der Tötung der Tiere, 3 Stdn. nach der Cortisolgabe kann daher auch nur auf einer primären Hemmung der Proteinsynthese beruhen.

Auf den Zusammenhang zwischen Enzyminduktion durch Cortisol und Gluconeogenese hatten schon HÜBENER und Mitarbeiter (8) hingewiesen. In letzter Zeit fanden StAIB und Mitarbeiter (9) bei Versuchen mit Aktinomycin D eine gute Korrelation zwischen Hemmung der Glykogensynthese in der Leber und Hemmung der Tyrosin-2-Oxoglutarat-TransaminaseInduktion. Die vorliegenden Untersuchungen zeigen, $\mathrm{da} B$ auch Barbital gleichzeitig mit Cortisol verabreicht, die Enzyminduktion und die Gluconeogenese im gleichen Ausmaß hemmt. Die Aktivität der Tryptophan- 
pyrrolase und der Tyrosin-2-Oxoglutarat-Transaminase sowie das Leberglykogen sind bei zusätzlicher Brabitalgabe nur halb so hoch wie bei alleiniger Cortisolgabe. Dieses Ergebnis ist nicht zuletzt in methodischer Hinsicht interessant, da Untersuchungen zur Cortisolgluconeogenese teilweise in langfristigen Barbiturat-Narkosen durchgeführt wurden $(21,22)$.

In diesem Zusammenhang muß erwähnt werden, daß von Barbital eine glykogenolytische Wirkung nicht bekannt ist (23). Nach wiederholten Gaben von Phenobarbital während mehrerer Tage fällt der Glykogengehalt bezogen auf $1 \mathrm{~g}$ Leberfrischgewicht nur etwa so viel ab, wie es der Protein- und RNA-Zunahme entspricht (24). Das Glykogen der Gesamtleber bleibt damit innerhalb mehrerer Tage praktisch unverändert. Die Threonindehydratase, als weiteres Beispiel für ein zu induzierendes Enzym bereitete uns zunächst einige Schwierigkeiten. Für unsere Fragestellung war eine kurzfristige und einheitliche Aktivitätszunahme erforderlich. Diese ließ sich weder durch eine einmalige Cortisolgabe noch durch Nahrungsentzug erzielen. $\mathrm{Zu}$ entsprechenden Ergebnissen kamen Schmidinger und KRÖGER (25) bei der Untersuchung der Serindehydratase. Es spricht vieles dafür, daß Serindehydratase und Threonindehydratase nur ein Enzymprotein sind (26, $27,28)$. Auch die Gabe von säurehydrolysiertem Casein erwies sich nicht sehr wirksam (Tab. 1) aufgrund des fehlenden Tryptophans (29).

Aus der Kombination von Nahrungskarenz mit zweimaliger Gabe von pankreatischem Caseinhydrolysat resultiert die beste, d.h. schnellste und einheitlichste Induktion der Threonindehydratase. Die Hemmbarkeit dieser Induktion durch Barbital (Abb. 4) zeigt erneut, daß die Induktionshemmung wie die Hemmung der Protein- und RNA-Synthese durch Barbital unabhängig von den Nebennierenrindenhormonen ist. Auch in diesem Modell ist Barbital noch zu einem Zeitpunkt wirksam, wenn Aktinomycin D die Induktion nicht mehr beeinflußt, sondern nur noch Puromycin (6). Das ist eine weitere Bestätigung der primären Hemmung der Proteinsynthese durch Barbital.

Peraino und Mitarbeiter (30) fanden bei ihren Untersuchungen über die Induktion der Threonindehydratase große Unterschiede, je nachdem, ob sie Phenobarbital nur einmal mit der ersten Caseindosis gaben oder ob sie die Tiere mehrere Tage mit Phenobarbital vorbehandelten. Diese Autoren vermuteten „Stoffwechseleffekte der akuten Phenobarbitalgabe"; aus den entsprechenden Diagrammen läßt sich eine Hemmung der Induktion von etwa $30 \%$ ablesen. Ein Hinweis dafür, daß zumindest Phenobarbital diesbezüglich eine ähnliche Wirkung wie Barbital hat.

\section{Literatur}

1. Kröner, H., B. Gutenberger, S. Hollmann und W. Statb, diese Z. 7, 8 (1969). - 2. KRöNER, H. und W. StArB, diese Z. 8, 41 (1970). - 3. Hollmand, S. und J. Neubaur, Hoppe Seyler's Z. physiol. Chem. 348, 877 (1967). - 4. KNox, W. E., Brit. J. exper. Path. 32, 462 (1951). - 5. KenNey, F. T. und R. M. FlorA, J. biol. Chemistry 236, 2699 (1961). - 6. Pitot, H. C. und C. Peraino, J. biol. Chemistry 239, 1783 (1964). - 7. CSANYI, V., O. GreENGARD und W. E. Knox, J. biol. Chemistry 242, 2688 (1967). - 8. Degenhardt, G., H. J. Hübener und J. Alester, Hoppe Seyler's Z. physiol. Chem. 323, 278 (1961). - 9. HerrmanN, J., G. A. Lauer und W. Starb, Hoppe Seyler's Z. physiol. Chem. 348, 1395 (1967). - 10. Pitot, H. C., V. R. Potrer und H. P. Morris, Cancer Res. 21, 1001 (1961). - 11. HAgEMANN, E. und G. Schmrdt, Ratte und Maus, Verlag de Gruyter und Co., Berlin (1960). 12. Farber, E., Cancer Res. 16, 142 (1956). - 13. Knox, W. E. und V. H. Auerbach, J. biol. Chemistry 214, 307 (1955). - 14. Ewald, W. und H. J. HÜBENER, Naturwissenschaften 48, 720 (1961). 15. GreENBERG, D. M., in: Methods in Enzymology Bd. V, S. 936, Edit. S. Colowick, N. O. Kaplan, Academic Press, New York, London (1962). - 16. Feigelson, P. und O. Greengard, J. biol.
Chemistry 237, 3714 (1962). - 17. KenNey, F. T., J. biol. Chemistry 237, 1610 (1962). - 18. KENNEY, F. T., J. biol. Chemistry 237, 3495 (1962). - 19. EwaLd, W., H. J. HÜBENER und E. WIEDemann, Hoppe Seyler's Z. physiol. Chem. 333, 57 (1963). - 20. Servelt, K. F., Acta Endocr. K'hvn Suppl. 88 (1963). - 21. Hornbrook, K. R., H. B. Busch und O.H. Lowry, Biochem. Biophys. Res. Comm. 18, 206 (1965). - 22. Young, D. A., Arch. Biochem. Biophysics 114, 309 (1966). - 23. Schaude, G., M. Siess, W. Vogell und G. Niessing, Acta Histochem. (Jena) 26, 185 (1967). - 24. Kunz, W., G. Schaude, H. Schimassek, W. Schmid und M. Sress, Proc. Europ. Soc. Study Drug Toxicity Vol. VII, 138 (1966). - 25. SchmIDINGER, H. und H. KRöger, Hoppe Seyler's Z. physiol. Chem. 348, 1367 (1967). - 26. Goldstenn I., W. E. Knox, E. J. Behrmann, J. biol. Chemistry 237, 2855 (1962). - 27. Freedland, R. A. und E. H. Avery, J. biol. Chemistry 239, 3357 (1964). - 28. Hoshino, J. und H. KRÖGER, Herbsttagung der Gesellschaft für Biologische Chemie, Münster, Oktober 1968. - 29. Peraino, C., R. L. Blake, H. C. Pitot, J. biol. Chemistry 240, 3039 (1965). - 30. Peraino, C., C. Lamar JR. und H. C. Prrot, J. biol. Chemistry 244, 2944 (1966).
Prof. Dr. S. Hollmann 4000 Düsseldorf 1 Witzelstr. 111 